\title{
Patrones de distribución de orquídeas en un relicto de bosque altoandino, Cundinamarca-Colombia
}

\section{Patterns of distribution of orchids in a high andean forest relict, Cundinamarca-Colombia}

\author{
Solmar David Califa Rodríguez ${ }^{10}$ y Luis Hernando Estupiñan Bravo ${ }^{\circledR}$
}

Califa, S.D. y Estupiñan, L.H. (2020). Patrones de distribución de orquídeas en un relicto de bosque altoandino, Cundinamarca-Colombia. Colombia forestal, 21(1), 5-19.

Recepción: 25 de abril 2019

\section{Resumen}

Colombia es uno de los países con mayor diversidad de orquídeas, cuenta con un estimado de 4270 especies nativas, de las cuales 199 presentan algún tipo de amenaza. Conocer las características que determinan su distribución es crucial para generar programas que propendan por su conservación. Este estudio buscó conocer la influencia de factores bióticos y abióticos sobre la distribución de orquídeas en un relicto de bosque altoandino en la región de Cundinamarca, Colombia, para tal fin se colectó información de las orquídeas encontradas en nueve transectos de $50 \mathrm{~m} \times 4 \mathrm{~m}$. Los factores ambientales que mayor influencia tuvieron sobre la abundancia y distribución de orquídeas fueron la altitud y el porcentaje de cobertura de musgos, así como las características inherentes a las comunidades de forófitos para el caso de las especies epífitas.

Establecer patrones de distribución de orquídeas permitirá, en el futuro, diseñar programas de conservación de esta flora en particular y del bosque altoandino en general.

Palabras clave: diversidad, forófito, Orchidaceae, porcentaje de cobertura de musgos.
Aprobación: 16 de diciembre 2020

\begin{abstract}
Colombia is one of the countries with greatest diversity of orchids, with 4270 native estimated species, of which 199 present some kind threat. Knowing the characteristics that determine their distribution is necessary to design conservation programs. This study aims to know the influence of biotic and abiotic factors on the distribution of orchids in a relict of high Andean forest in the region, of Cundinamarca, Colombia, for this purpose information was collected on the orchids found in nine transects of $50 \mathrm{~m} \times 4 \mathrm{~m}$. The environmental factors that had the greatest influence on the distribution of orchids were the altitude and the percentage of moss cover, as well as the characteristics inherent to the communities of forophytes, in the case of epiphytic species.

Establishing orchid distribution patterns will allow, in the future, to design conservation programs for this particular flora and the high Andean forest in general.
\end{abstract}

Keywords: diversity, phorophyte, Orchidaceae, percentage of moss cover. 


\section{INTRODUCCIÓN}

Colombia es uno de los países con mayor diversidad de orquídeas en el mundo, con alrededor de 4270 especies en 274 géneros (Betancur, Sarmiento, Toro-Gonzáles y Valencia, 2015). Esta diversidad es explicada por la alta heterogeneidad de hábitats que se encuentran en el territorio nacional debido a su topografía quebrada y discontinua (Giraldo y Betancur, 2011). Sin embargo, 199 especies se encuentran en alguna categoría de amenaza (Betancur et al., 2015).

La pérdida de hábitat, fragmentación de los bosques y alteraciones de las condiciones originales en los ecosistemas son enumeradas como las principales amenazas que se ciernen sobre la familia Orchidaceae; sumándose a estas amenazas la sobrecolecta y comercio ilegal de orquídeas que afecta a taxones caracterizados por ser utilizados por comunidades humanas en diversas actividades (IUCN/SSC Orchid Specialist Group, 1996; Solano, Cruz, Martinez y Lagunez, 2010; Orejuela-Gartner, 2012).

En Colombia se han realizado inventarios que abordan de manera adecuada y sistemática la flora orquidácea del país (Ortiz y Uribe-Vélez, 2007; Calderón, 2007; Betancur et al., 2015) y trabajos destinados a conocer la influencia de factores abióticos y bióticos sobre distribución de orquídeas (Parra, 2012; Mejía y Pino, 2010; Santa-Jiménez, García y Gómez 2009). Sin embargo, se carece de estudios encaminados a conocer el estado de sus poblaciones, e inclusive la percepción que tienen las comunidades humanas sobre este grupo de plantas y el papel que juegan en su conservación.

Alrededor del $73 \%$ de las especies de orquídeas son epífitas (Atwood, 1986). Según Otero, Aragón y Ackerman (2007), se han observado variaciones importantes en los patrones de distribución de epífitas.

La presencia de una población de plantas en un área determinada significa que sus requerimientos se encuentran de una u otra manera satisfechos, situación en la que los factores abióticos y bióticos determinan el potencial de crecimiento y reproducción de una especie en particular (Davis et al., 1990; Davis y Heywood, 1994; Ibisch, Nowicki, Müller y Araujo, 2002). Igualmente, factores abióticos como la humedad atmosférica, la precipitación, la radiación solar y la altura sobre el nivel del mar condicionan en gran medida la abundancia y diversidad de epífitas (Sugden y Robins, 1979; Davis et al., 1990; Davis y Heywood, 1994; Ding et al., 2016).

Los patrones de distribución y agregación de epífitas se ven influenciados por los distintos micro climas que se evidencian en los diferentes estratos que se encuentran dentro de un ecosistema, donde los grados de radiación (densidad de flujo fotónico) y humedad varían (De la Rosa-Manzano et al., 2014). La disponibilidad de la luz caracteriza los micrositios de los forófitos habitados por orquídeas epífitas (Ventre-Lespiaucq et al., 2017). De esta manera, factores como la densidad de forófitos en un área y sus tipos de arquitectura (altura, inclinación, grosor del tronco y número, grosor, distribución y posición de las ramas) influyen sobre la distribución de epífitas, no solo por las consecuencias que estos factores tienen sobre la acumulación de humedad, la velocidad del viento y el paso de luz, sino también por las posibilidades que pueden brindar para el depósito de semiIlas y materia orgánica (Wagner, Mendieta-Leiva y Zotz, 2015); además de ello, factores específicos del forófito como la textura, porosidad, $\mathrm{pH}$, composición química de la corteza, y la presencia líquenes, musgos y otras plantas también influencian la distribución de plantas epífitas (Cornelissen y ter Steege, 1989).

Las orquídeas son exigentes en sus requerimientos de sustratos $\mathrm{u}$ hospederos, polinizadores, hongos micorrícicos, además de ser muy específicas en cuanto a condiciones climáticas se refiere (Calderón, 2007; Orejuela, 2015); por lo tanto, el aumento en el conocimiento de aquellos factores bióticos y abióticos que determinan la distribución y que condicionan el desarrollo de las orquídeas en su hábitat natural permite generar o mejorar las estrategias 
existentes para su reintroducción y conservación in situ, no solo en aquellas regiones estudiadas, sino también en regiones con similitudes climáticas, geográficas y florísticas, posibilitando además emitir alertas tempranas en aquellos ecosistemas en los que se detecten cambios en estas condiciones.

Con la finalidad de conocer la influencia de los factores bióticos y abióticos sobre la distribución de orquídeas en un relicto de bosque altoandino ubicado en el distrito de manejo integrado de los recursos naturales Salto del Tequendama-Manjui, la presente investigación buscó resolver las siguientes preguntas: ¿existen patrones de distribución de orquídeas en el fragmento de bosque alto andino a estudiar? y ¿qué características particulares de hábitat/forófito-sustrato o sustrato están asociadas con la presencia o ausencia de orquídeas?

\section{MATERIALES Y MÉTODOS}

\section{Área de estudio}

El estudio se realizó en un relicto de bosque altoandino que forma parte del orobioma andino vertiente occidental de la Cordillera Oriental entre los 2770 y los 3020 m de altitud, ubicado en distrito de manejo integrado de los recursos naturales Salto del Tequendama-Manjui Cundinamarca, Colombia. Con la finalidad de conocer la extensión del relicto de bosque y sus particularidades geográficas y climáticas, se realizó el reconocimiento visual de coberturas vegetales por medio de la observación y procesamiento de un conjunto de imágenes del servicio Google Earth, Digital Globe para, posteriormente, digitalizar polígonos correspondientes al relicto de bosque seleccionado y las curvas de nivel de la zona a estudiar, por medio

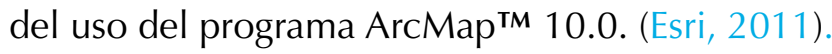

\section{Factores ecológicos}

Se realizaron salidas de campo entre los meses de octubre del 2017 a marzo del 2018, periodo en el que se establecieron un total de 9 parcelas de 50 $\mathrm{m} \times 4 \mathrm{~m}\left(200 \mathrm{~m}^{2}\right)$ distribuidas aleatoriamente en un relicto de bosque dentro del distrito de manejo. Se realizaron tres visitas por transecto, cada una de aproximadamente seis horas.

En cada transecto se realizó el inventario del total de las especies de orquídeas epífitas y terrestres presentes; además, se registraron los siguientes datos para orquídeas epífitas: estado del forófito (vivo o muerto), orientación de la rama o tronco (horizontal, vertical o inclinada), tipo de corteza del forófito (lisa o rugosa), porcentaje de cobertura de musgos, distribución vertical según las zonas de Johansson (1974) y diámetro a la altura del pecho (dap). Para las orquídeas terrestres se tomó la profundidad del mantillo en el que se encontraron.

Se tomaron fotografías digitales de abajo hacia arriba del dosel por cada zona, de acuerdo con las establecidas por Johansson (1974), en las que se encontraron orquídeas epífitas o por cada sitio en el que se encontraron orquídeas terrestres; se prestó atención especial a que los rayos del sol no incidieran de manera directa en el lente de la cámara. Por medio del programa GLA® Gap Light Analyzer (Forest Renewal Bc, 1999) se analizaron las fotografías, considerando el brillo solar y las coordenadas geográficas de la reserva, para obtener el porcentaje de apertura del dosel como una medida indirecta de la radiación lumínica.

Para realizar la evaluación hábitat-forófito-sustrato se determinó inicialmente del diámetro a la altura del pecho (dap) del forófito a $1.3 \mathrm{~m}$ de la superficie del suelo utilizando cinta diamétrica. En el caso de bifurcación de las ramas se procedía en forma individual en cada una de las bifurcaciones a una altura de $1 \mathrm{~m}$. Por su parte, el diámetro de la copa se estimó midiendo dos diámetros perpendiculares de la copa sobre el suelo (Andrade y Cerda, 2010).

El porcentaje de cobertura de líquenes o musgos presentes en el tronco y en las ramas en las que se hospedan las orquídeas se determinó de acuerdo con los siguientes porcentajes: $0 \%, 25 \%, 50 \%$, $75 \%$ y $100 \%$ (Gowland et al., 2011). 
Para la identificación de orquídeas se tomaron fotografías de cada una de las morfoespecies y se registró información morfológica en formatos diseñados para tal fin. Cuando se encontraron plantas en estado fértil se colectaron de 1 a 4 flores, las cuales se almacenaron en una mezcla de alcohol y glicerina buscando preservar sus estructuras y la coloración de estas. Especímenes que no se identificaron en campo fueron ubicados en el orquideario de la Universidad de Ciencias Aplicadas y Ambientales (UDCA) esperando a su floración, ello con el objetivo de identificarlos taxonómicamente. Es importante mencionar que la UDCA cuenta con el Permiso Marco de Recolección de especímenes silvestres de diversidad biológica con fines de investigación científica de acuerdo con la resolución 1138 del 15 de noviembre de 2013 de la Autoridad Nacional de Licencias Ambientales (Anla) y permiso de la CAR otorgado para el proyecto "Identificación y evaluación de las poblaciones de orquídeas de la estructura principal de Bogotá y los cerros adyacentes a la sabana de Bogotá con fines de conservación", del cual hace parte la presente investigación. En ningún caso se colectaron y procesaron especímenes para ser depositados en algún herbario.

La identificación de las morfoespecies se realizó con las siguientes claves taxonómicas: Luer (1986); Ortiz (1995); Hammel, Mendieta-Leiva y Zotz (2003). Las series Icones Orchidacearum (Hágsater, 2016; 2009; 2001); Icones Pleurothallidinarum (Luer, 1986; 2004; Luer y Thoerle, 2012) y la Galería de Orquídeas de Colombia (Ortiz y Uribe-Vélez, 2007). Se utilizaron, además, las bases de datos de Pfahl (2018) y del Missouri Botanical Garden (2018), los herbarios en línea del Instituto de Ciencias Naturales de la Universidad Nacional de Colombia (2018), Jardín Botánico de Bogotá José Celestino Mutis (2018) y de la Swiss Orchid Foundation (2016).

La información se organizó en hojas de cálculo de LibreOffice Calc 5.1.6.2 (Colaboradores de LibreOffice, 2016), se realizaron tablas de frecuencia y gráficas con el propósito de analizar la información. A partir de la tabla general de abundancias se obtuvieron los índices de diversidad de Shannon y Dominancia de Simpson, además de dendrogramas mediante el cálculo de los índices de afinidad de Morisita y Jaccard. Se realizó el análisis de correspondencia canónica (ACC) para las orquídeas epífitas y terrestres, para lo cual se transformaron con logaritmo los datos ambientales y del forófito; estos análisis se realizaron utilizando el paquete de análisis de datos, PAST "Paleontological Statistics Software Package for Education and Data Analysis Statistical Package" (Hammer et al., 2001).

\section{RESULTADOS}

Se registraron un total de 1064 individuos en un área total de $1800 \mathrm{~m}^{2}$, de los cuales 779 tuvieron un hábito epífito, 11 litofito y 274 terrestre.

Se identificaron 55 morfoespecies (tablas 1 y 2), 16 a nivel de especie, 24 a nivel de género (Cyrtochilum Kunth, Elleanthus CPresl, Epidendrum L, Fernandezia Ruiz \& Pav., Gomphichis Lindl., Stelis Sw y Telipogon Kunth) y 15 a nivel de subtribu y tribu (Laeliinae Benth, Oncidiinae Benth, Pleurothallidinae Lindl. ex G. Don y Sobralieae Pfitzer). Los valores para toda el área estudiada del índice de dominancia de Simpson y de Shannon fueron de 0.1 y 2.98 , respectivamente.

Las especies más abundantes fueron Acronia phalangifera, Stelissp. 1 y Epidendrumcalyptratoides (tablas 1 y 2), siendo E. calyptratoides la especie encontrada con más frecuencia en los transectos (seis transectos), seguida de $A$. phalangifera, Epidendrum sp. 1, Epidendrum megalospathum y Oncidiinae 1, encontradas todas en tres de los transectos.

Las especies registradas fueron encontradas dentro de los transectos evaluados. Cabe mencionar que dentro del distrito de manejo y por fuera de los transectos se observaron las siguientes especies: Cyrtochilum annulare (Rchb. F.) Kraenzl., 
Tabla 1. Morfoespecies de orquídeas epífitas encontradas en los transectos ubicados en el relicto del bosque altoandino, Cundinamarca, Colombia. (T) transecto, (A) Abundancia, (mat) matorral

\begin{tabular}{|c|c|c|c|c|}
\hline Morfoespecie & Altitud (m) & $\mathbf{T}$ & A & Zonas \\
\hline Acianthera casapensis (Lindl.) Pridgeon \& M.W. Chase & $2851-2949$ & T1, T5 & 10 & II, III \\
\hline Acronia phalangifera C. Presl & $2851-2949$ & $\mathrm{~T} 2, \mathrm{~T} 3, \mathrm{~T} 5$ & 282 & I, II, III \\
\hline Crocodeilanthe floriibunda (Poepp. \& Endl.) Luer & 2929-3018 & T3, T9 & 8 & I, II \\
\hline Cyrtochilum sp1 & $2880-2887$ & T4 & 5 & II, III \\
\hline Cyrtochilum sp 2 & 2851 & T5 & 23 & III, IV \\
\hline Epidendrum calyptratoides Hágsater \& Dodson & $2767-2945$ & $\begin{array}{c}\mathrm{T} 2, \mathrm{~T} 4, \mathrm{~T} 5, \mathrm{~T} 6, \\
\mathrm{~T} 7, \mathrm{~T} 8\end{array}$ & 80 & I, II, III, IV \\
\hline Epidendrum megalospathum Rchb. f. & $2779-2876$ & T5, T6, T8 & 14 & II, III \\
\hline Epidendrum sp9 & 3018 & T9 & 1 & mat \\
\hline Epidendrum sp10 & 2891 & $\mathrm{~T} 4$ & 1 & II \\
\hline Epidendrum sp11 & 2883 & $\mathrm{~T} 2$ & 10 & II, III, IV \\
\hline Epidendrum sp12 & 2918-3018 & $\mathrm{T} 3, \mathrm{~T} 9$ & 2 & III, mat \\
\hline Epidendrum sp13 & 2910 & $\mathrm{~T} 1$ & 1 & II \\
\hline Fernandezia sp & 2949 & $\mathrm{~T} 1$ & 1 & IV \\
\hline Laeliinae 1 & 2773 & T7 & 2 & $\mathrm{tm}$ \\
\hline Laeliinae 2 & 2851 & T5 & 11 & 1 \\
\hline Lepanthes cornualis Luer \& R. Escobar & $2851-2934$ & $\mathrm{~T} 3, \mathrm{~T} 5$ & 75 & II, III \\
\hline Lepanthes troxis Luer \& R. Escobar & 2918-2934 & T3 & 51 & II, III \\
\hline Maxillaria aggregata (Kunth) Lindl. & 2931-3018 & T3, T9 & 18 & II \\
\hline Oncidiinae 1 & $3825-2918$ & $\mathrm{~T} 3, \mathrm{~T} 4, \mathrm{~T} 5$ & 9 & II, III \\
\hline Oncidiinae 2 & $2870-2886$ & T6 & 39 & II, III, IV \\
\hline Oncidiinae 3 & 2949 & $\mathrm{~T} 1$ & 4 & II \\
\hline Oncidiinae 4 & $2918-2926$ & T3 & 9 & II, III \\
\hline Oncidiinae 5 & $2910-2949$ & $\mathrm{~T} 1$ & 3 & III, V \\
\hline Oncidiinae 6 & 2851 & T5 & 38 & II \\
\hline Oncidiinae 7 & $2767-2787$ & T7 & 32 & I, II, III, IV \\
\hline Oncidium cultratum Lindl. & 2918 & T3 & 1 & III \\
\hline Oncidium ornithorhynchum Kunth & $2767-2851$ & $\mathrm{~T} 5, \mathrm{~T} 7$ & 3 & IV \\
\hline Pleurothallidinae 1 & 2918 & T3 & 1 & II \\
\hline Pleurothallidinae 2 & 2932 & $\mathrm{~T} 3$ & 7 & I, II \\
\hline Pleurothallidinae 3 & 2769 & T8 & 3 & III \\
\hline Pleurothallidinae 4 & 3018 & T9 & 8 & mat \\
\hline Stelis sp & $2918-2931$ & $\mathrm{~T} 3$ & 100 & I, II, III \\
\hline Stelis sp2 & 2918-2934 & $\mathrm{T} 3$ & 28 & $\mathrm{I}, \mathrm{II}$ \\
\hline
\end{tabular}

Fuente: elaborado según clasificación de Johansson (1974).

Elleanthus aurantiacus (Lindl.) Rchb. F., Odontoglossum gloriosum Linden \& Rchb. F. y Pleurothallis talpinaria Rchb. f.).

Es importante mencionar que un $74.5 \%$ de las morfoespecies se encontraron restringidas a un solo sitio de muestreo.

\section{Transectos}

Los transectos con mayor abundancia de orquídeas fueron T3, T2 y T5. Si bien cambian su composición estos comparten la presencia de A. phalangifera, T2 y T5 comparten E. calyptratoides, y, T3 
Tabla 2. Orquídeas terrestres encontradas en los transectos ubicados en un relicto del bosque altoandino, Cundinamarca, Colombia. (trans) transecto, (Abun) abundancia

\begin{tabular}{cccc}
\hline Morfoespecie & Altitud $(\mathbf{m})$ & trans & Abun \\
\hline Acronia phalangifera C. Presl & $2851-2949$ & $\mathrm{~T} 2, \mathrm{~T} 3$ & 90 \\
Cyrtochilum sp & 2910 & $\mathrm{~T} 1$ & 1 \\
Elleanthus maculatus (Lindl.) Rchb. f. & 2918 & $\mathrm{~T} 3$ & 5 \\
Elleanthus sp & 3018 & $\mathrm{~T} 9$ & 26 \\
Epidendrum decurviflorum Schltr. & 3018 & $\mathrm{~T} 9$ & 15 \\
Epidendrum sp1 & $2910-3018$ & $\mathrm{~T} 1, \mathrm{~T} 2, \mathrm{~T} 9$ & 28 \\
Epidendrum sp2 & 3018 & $\mathrm{~T} 9$ & 11 \\
Epidendrum sp3 & 2918 & $\mathrm{~T} 3$ & 22 \\
Epidendrum sp4 & 3018 & $\mathrm{~T} 9$ & 2 \\
Epidendrum sp5 & 2874 & $\mathrm{~T} 6$ & 7 \\
Epidendrum sp6 & 2910 & $\mathrm{~T} 1$ & 4 \\
Epidendrum sp7 & 3018 & $\mathrm{~T} 9$ & 5 \\
Epidendrum sp8 & 3018 & $\mathrm{~T} 9$ & 9 \\
Epidendrum sp10 & 3018 & $\mathrm{~T} 9$ & 12 \\
Gomphichis sp & $2776-2880$ & $\mathrm{~T} 4, \mathrm{~T} 8$ & 1 \\
Oncidinae 8 & 3018 & $\mathrm{~T} 9$ & 2 \\
Pleurothallidinae 4 & 3018 & $\mathrm{~T} 9$ & 3 \\
Sobraliinae 1 & 2918 & $\mathrm{~T} 3$ & 2 \\
Telipogon sp & 2910 & $\mathrm{~T} 1$ & 7 \\
\hline
\end{tabular}

y T5 L. cornualis, especies que se caracterizaron por presentar los mayores valores de abundancias, $\mathrm{n}=282, \mathrm{n}=80$ y $\mathrm{n}=75$, respectivamente (tabla 1 ). Asimismo, en T5 se encuentra la mayor abundancia de Oncidiinae $(n=66)$, mientras en T2 no se encuentran plantas de esta subtribu.

Los mayores valores del índice de Simpson fueron para T2, T8 y T7, en estos una de sus especies fue claramente dominante pues su abundancia relativa fue mayor al $60 \%$ (A. phalangifera en el T2 con un $90.85 \%$, Oncidiinae 7 en el T7 con $66.6 \%$ y Epidendrum calyptratoides en T8 con $62.96 \%$ ), siendo de igual forma los transectos con menores índices de Shannon (tabla 3).

En cuanto a riqueza respecta $\mathrm{T} 9$ fue el transecto con mayor número de morfoespecies, seguido de T3 y T5. Los transectos en los que se registraron la mayor cantidad de morfoespecies únicas fueron T9, T3 y T1, teniendo 11, 10 y 7 morfo especies restringidas, respectivamente.

Tabla 3. Valores de abundancia, riqueza y diversidad de orquídeas en los transectos trazados en el relicto de bosque altoandino, Cundinamarca, Colombia. Altitud en m (A) Abundancia de orquídeas, (R) Riqueza de orquídeas, (D) índice de dominancia de Simpson, $\left(\mathrm{H}^{\prime}\right)$ índice de diversidad de Shannon

\begin{tabular}{ccccccccccc}
\hline & T1 & T2 & T3 & T4 & T5 & T6 & T7 & T8 & T9 & Total \\
\hline altitud & 2923 & 2928 & 2924 & 2869 & 2851 & 2876 & 2773 & 2778 & 3018 & - \\
A & 26 & 175 & 367 & 38 & 167 & 76 & 48 & 27 & 140 & 1064 \\
R & 9 & 4 & 16 & 5 & 10 & 4 & 4 & 4 & 17 & 52 \\
$\mathbf{D}$ & 0.16 & 0.83 & 0.18 & 0.33 & 0.17 & 0.38 & 0.52 & 0.57 & 0.10 & 0.10 \\
$\mathbf{H}^{\prime}$ & 1.97 & 0.37 & 1.99 & 1.28 & 1.94 & 1,07 & 0.83 & 1.06 & 2.47 & 2.98 \\
\hline
\end{tabular}


El transecto 9 presentó la mayor diversidad de orquídeas según el índice de Shannon, vale la pena acotar que un $64 \%$ de las morfoespecies del transecto 9 pertenecen al género Epidendrum, de las cuales varias son terrestres, por lo que la composición de este transecto difiere respecto a los demás sitios de muestreo.

Acronia phalangifera $(\mathrm{n}=282)$ y Epidendrum calyptratoides $(\mathrm{n}=80)$ son las especies más abundantes y de distribución más amplia en este estudio, debido a que ocuparon diversos tipos de forófitos. Sin embargo, un alto porcentaje de las especies encontradas se halló en condiciones ambiente/forófito específicas.

\section{Variables ambientales}

Los dos primeros ejes del ACC para epífitas (figura 1) representan el $83.45 \%$ de la variación entre las especies y las condiciones ambientales. El ACC para especies terrestres (figura 2) muestra en sus dos primeros ejes el $100 \%$ de la variación.

Existen tres conjuntos de variables relacionadas con la presencia de orquídeas epífitas en los transectos:

- Aquellas relacionadas con la calidad del forófito: el porcentaje de cobertura de musgos, el dap y la corteza, las cuales explican gran parte de

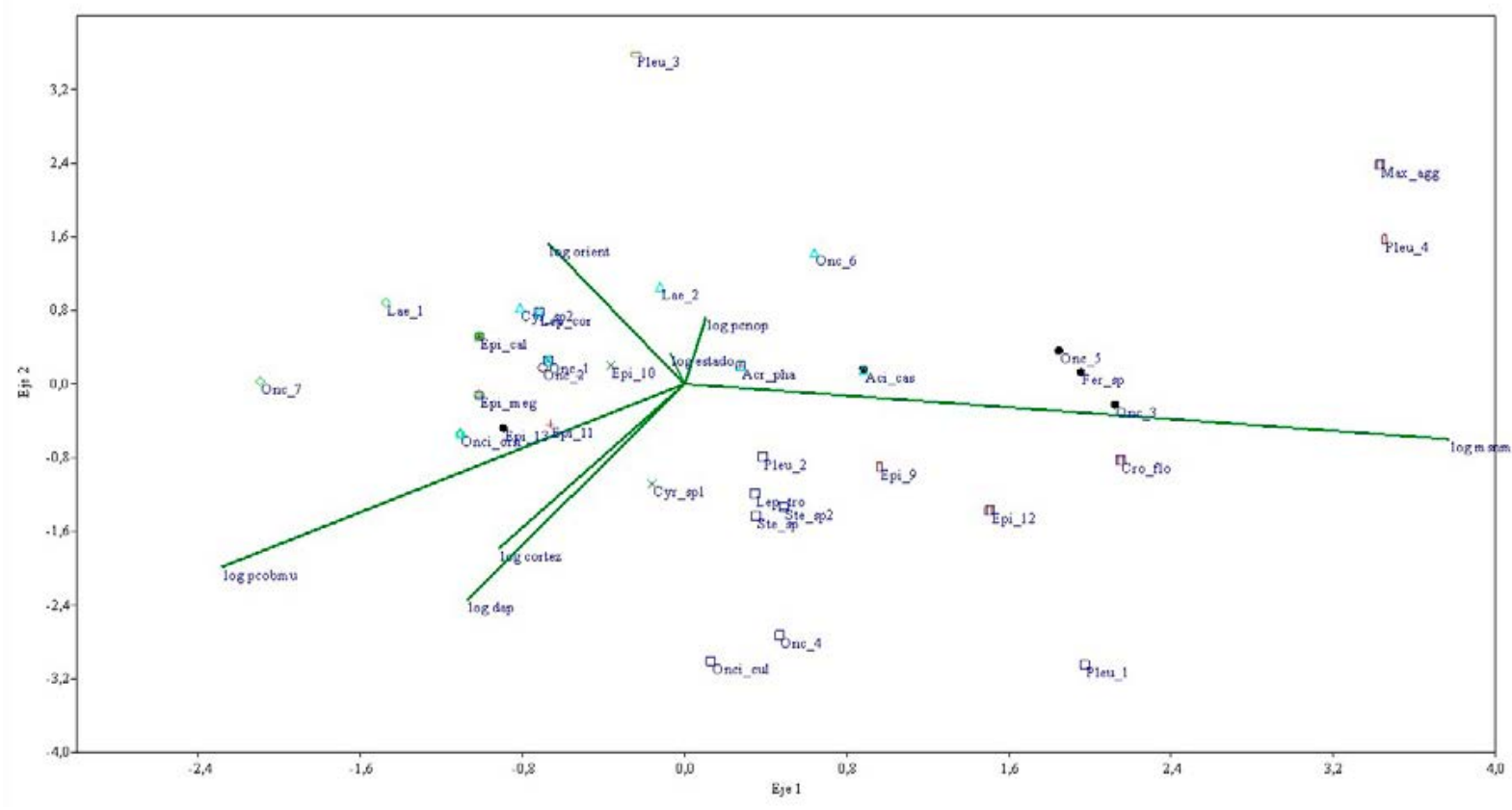

Figura 1. ACC que muestra las morfoespecies de orquídeas epífitas y su relación con el logaritmo de variables hábitat/forófito. (log msnm) metros sobre el nivel del mar, (orienta) orientación, (\%musgos) Porcentaje de cobertura de musgos, (corteza) corteza forófito. Especies (Aci_cas) Acianthera casapensis, (Acr_phal) Acronia phalangifera, (Cro_flo) Crocodeilanthe floribunda, (Cyr_sp1) Cyrtochilum sp1, (Cyr_sp2) Cyrtochilum sp2, (Epi_10) Epidendrum sp10, (Epi_11) Epidendrum sp11, (Epi_12) Epidendrum sp12, (Epi_13) Epidendrum sp13, (Epi_9): Epidendrum sp9, (Epi_cal) Epidendrum calyptratoides, (Epi_meg) Epidendrum megalospathum, (Fer_sp) Fernandezia sp, (Lae_1) Laeliinae 1, (Lae_2) Laeliinae 2, (Lep_cor) Lepanthes cornualis, (Lep_tro) Lepanthes troxis, (Max_agg) Maxillaria aggregata, (Onc_1) Oncidiinae 1, (Onc_2) Oncidiinae 2, (Onc_3) Oncidiinae_3, (Onc_4) Oncidiinae 4, (Onc_5) Oncidiinae 5, (Onc_6) Oncidiinae 6, (Onc_7) Oncidiinae 7, (Onci_cul) Oncidium cultratum, (Onci_orn) Oncidium ornithorhynchum, (Pleu_1) Pleurothallidinae 1, (Pleu_2) Pleurothallidinae 2, (Pleu_3) Pleurothallidinae 3, (Pleu 4) Pleurothallidinae 4; (Ste_sp) Stelis sp, (Ste_sp2) Stelis sp2. 
la variación. Teniendo el porcentaje de cobertura de musgos los mayores valores en los ejes 1 y 2 , seguido del dap (diámetro a la altura del pecho) y la corteza. Este grupo de vectores son opuestos al vector de altura.

- Variables relacionadas con la arquitectura del forófito, la orientación, el estado del forófito y el porcentaje de apertura del dosel, en la cual el vector de orientación tiene la mayor longitud.

- La altura sobre el nivel del mar, vector que tiene la mayor longitud de los vectores representados y al que se asocian varias especies de orquídeas epífitas.

En el caso de las especies terrestres se realizó el ACC con el porcentaje de apertura del dosel, la altura sobre el nivel del mar y la profundidad del sustrato (figura 2). Siendo la apertura del dosel y la altura sobre el nivel del mar los vectores que tuvieron una mayor fuerza.

La combinación de estas variables forófito/ambiente posibilita la presencia de ciertos grupos de orquídeas, encontrándose orquídeas que se desarrollan ya exclusiva o preferentemente en atributos precisos del forófito o en condiciones ambientales muy definidas, mientras otras tienden a ser generalistas. En los análisis de ordenación de las especies de orquídeas se pueden reconocer cinco grupos, tres de especies epífitas y dos de especies terrestres. A continuación, se describe cada uno:

- Grupo 1: especies epífitas que se encuentran generalmente por debajo de los $2900 \mathrm{~m}$ de altitud (T4, T5, T6, T7 y T8) con diferente nivel de asociación al porcentaje de cobertura de musgos, en forófitos con porcentaje de cobertura de musgos

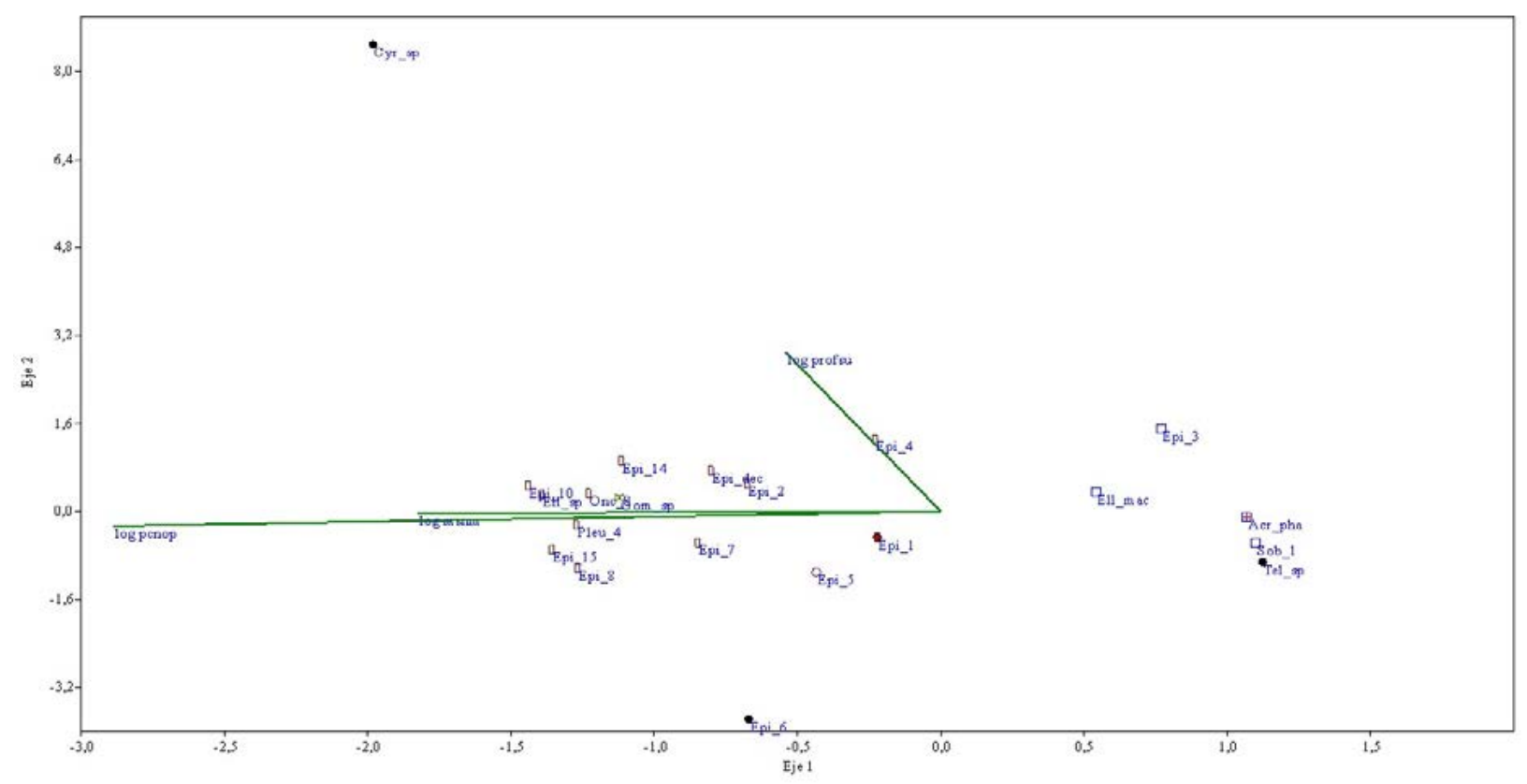

Figura 2. ACC que muestra las morfoespecies de orquídeas epífitas y su relación con el logaritmo de variables de hábitat. (profsus) profundidad del sustrato, (\%apedo) porcentaje de apertura del dosel. Especies: (Acr_phal) Acronia phalangifera, (Cyr_sp) Cyrtochilum sp, (Elle_mac) Elleanthus maculatus, (Elle_sp) Elleanthus sp, (Epi_sp1) Epidendrum sp1, (Epi_10) Epidendrum sp10, (Epi_14) Epidendrum sp14, (Epi_15) Epidendrum sp15, (Epi_12) Epidendrum sp12, (Epi_3) Epidendrum sp3, (Epi_4) Epidendrum sp4, (Epi_5) Epidendrum sp5, (Epi_6) Epidendrum sp6, (Epi_7) Epidendrum sp7, (Epi_8): Epidendrum sp8, (Epi_dec): Epidendrum decurviflorum, (Gom_sp): Gomphichis sp, (Onc_8) Oncidiinae 8, (Pleu_4) Pleurothallidinae 4, (Sob_1) Sobraliinae 1, (Tel_sp) Telipogon sp. 
media a alta y dap promedio por morfoespecie entre $0.34 \mathrm{~cm}$ (Oncidiinae 2) y $14.4 \mathrm{~cm}$ (Oncidiinae 7), con preferencia por forófitos de orientación vertical.

Se destacan dentro de este grupo Laeliinae 1 y Cyrtochilum sp. 2, Oncidiinae 1 y E. megalospathum al encontrarse exclusivamente en forófitos de corteza lisa.

- Grupo 2: especies epífitas que usualmente se encuentran por encima de los 2900 m de altitud (T3 y T9) halladas en forófitos con valores medios a altos del porcentaje de cobertura de musgos y dap promedio por especie entre $4.77 \mathrm{~cm}$ (Epidendrum sp. 9) y $24.83 \mathrm{~cm}$ (Oncidium cultratum), estas especies presentan diferente nivel de asociación con el dap. La mayoría de ellas se diferenciaron por su preferencia por forófitos con orientación vertical, mientras Epidendrum sp12 y Oncidiinae 4 se encontraron en forófitos con orientación inclinada y Crocodeilanthe floribunda en forófitos con orientación horizontal, inclinada y vertical.

Oncidiinae 4 creció exclusivamente en árboles de corteza lisa, mientras las demás especies no tuvieron preferencia por algún tipo de corteza o se encontraron en un único forófito.

- Grupo 3: especies epífitas, asociadas al gradiente de altura sobre el nivel del mar (encontradas en los transectos T1, T2, T3 y T9) y sin preferencia por la orientación (creciendo en matorrales, troncos muertos, árboles muertos y árboles) encontradas generalmente en forófitos con dap promedio por especie bajo (inferior a los $10 \mathrm{~cm}$ ), usualmente en forófitos con niveles bajos de cobertura de musgos, con una baja asociación al porcentaje de apertura del dosel, en la cual el promedio por especie varió desde el $6.3 \%$ (para A. casapensis) al $30.23 \%$ (para Oncidinae 5). Las especies de $\mathrm{T} 1$ se encuentran en este grupo con excepción de Epidendrum sp.13 que se encontró en un forófito con alto porcentaje de cobertura de musgo.
Se destacan Pleurothallidinae 4 que creció únicamente en forófitos de corteza rugosa, mientras M. aggregata y Oncidiinae 6 se encontraron en forófitos de corteza lisa.

A. phalangifera es la especie más generalista de este grupo pues se encontró en ramas con todos los tipos de orientación y en plantas con dap que variaron desde los $2.55 \mathrm{~cm}$ hasta los $24.5 \mathrm{~cm}$.

Se halló una estrecha relación entre el gradiente altitudinal y la riqueza de orquídeas epífitas.

Grupo 4: especies terrestres asociadas en diferente grado a la altura sobre el nivel del mar y al porcentaje de apertura del dosel, distribuidas en lugares con valores de apertura del dosel superiores al $10 \%$, generalmente por encima de los 2900 m de altitud, la mayoría en T9.

En este grupo se encuentra Gomphichis sp. registrada a los 2880 y 2776 m de altitud, especie muy asociada al vector de porcentaje de apertura del dosel.

- Grupo 5: especies terrestres que se encontraron porcentajes inferiores al $10 \%$ de apertura del dosel, profundidad variable y alturas menores a las del grupo 4. Estas especies pertenecen a los transectos T1, T2 y T3.

\section{DISCUSIÓN}

Las comunidades de orquídeas observadas dependen de la compleja interrelación entre factores ecológicos, las comunidades de forófitos establecidas en el área, sus características, su dispersión y heterogeneidad; además de los factores históricos que se expresan en términos de estabilidad de los ecosistemas y en los niveles de disturbio al que estos han estado sometidos (Baadi, Landeros y Cerna, 2008).

Se observaron patrones de distribución con respecto a la especie; sin embargo, algunas especies del mismo género tendieron a encontrarse en lugares que comparten condiciones similares, como es el caso de dos especies de Stelis evaluadas. Especies de Lepanthes se registraron por encima de 
los 2900 m de altitud; no obstante, L. cornualis se registró por debajo de los $2900 \mathrm{~m}$ de altitud y muestra una mayor dependencia del porcentaje de cobertura de musgos. Por su parte, L. troxis mostró una mayor relación con el dap.

E. megalospathum y E. calyptratoides (ambas del grupo Megalospathum) crecieron en lugares con porcentajes de cobertura de musgos medios a altos por debajo de los $2900 \mathrm{~m}$ de altitud. Sin embargo, el nivel de dependencia a las variables del forófito es mayor en E. megalospathum (que solo crece en forófitos con corteza lisa y de orientación vertical), lo que restringe su distribución en mayor medida que la de E. calyptratoides. Donde mejor se puede observar este comportamiento es en el complejo de especies terrestres de Epidendrum (grupo 4), pues estas se encontraron en lugares por encima de los $2870 \mathrm{~m}$ de altitud diferenciándose los sitios en los que se encontró cada morfoespecie al poseer porcentajes de apertura del dosel diferentes.

En este estudio se encontró asociación entre la distribución de orquídeas y la altura sobre el nivel del mar, en el intervalo considerado de 2770 a $3020 \mathrm{~m}$ de altitud y coincide con lo reportado por algunos autores que hallaron una estrecha relación entre el gradiente altitudinal y la riqueza de orquídeas epífitas como: Roberts y Brummitt (2006) en las islas Mascareñas, Yulia, Budiharta y Yulistyarini (2011) en Mount Lawu en Java, Focho, Fonge, Fongod y Essomo (2010) en Camerún, Tian y Xiang (2008) en la reserva natural Nanling en China y Revilla, Moscos y Nauray (2003) en Cuzco, Perú. Estos autores observaron, además, que a ciertas elevaciones se dan los mayores valores de diversidad de orquídeas los cuales disminuyen a medida que la elevación aumenta o disminuye, las elevaciones en las cuales se encuentran los picos de diversidad son diferentes en cada uno de los estudios citados, lo que demostraría que estas a su vez son dependientes de condiciones asociadas a su ubicación.

Los efectos de la altura en la diversificación de las orquídeas pueden deberse a la diversidad de micrositios que se originan en las zonas montañosas, característica que poseen muchas de las zonas neotropicales, lo que explica en parte la gran diversidad de orquídeas que se encuentran en esta área. Gentry y Dodson (1987) señalan además que los efectos de la altura y la humedad sobre las epífitas se encuentran complejamente interrelacionados.

No se encontró, como se muestra en la figura 2, una relación estrecha entre el porcentaje de apertura del dosel como medida indirecta de la radiación lumínica y la abundancia de orquídeas epífitas. De igual forma, Tremblay (2008) en un intento por medir el efecto del porcentaje de cobertura del dosel sobre las poblaciones de Lepanthes eltoroensis no encontró relación directa o cuadrática entre la proporción de luz y el número de individuos de esta especie. Sin embargo, sí se encontró una relación estrecha entre la abundancia de orquídeas terrestres y el gradiente de apertura del dosel, lo que podría indicar que las orquídeas terrestres ocupan nichos lumínicos que no son ocupados por las especies epífitas.

El porcentaje de cobertura de musgos mostró ser una de las variables que condicionó en gran medida el establecimiento de las especies de orquídeas epífitas encontradas, la asociación o presencia de otros organismos epífitos podría condicionar de forma directa o indirecta el establecimiento de orquídeas en determinadas zonas. De esta manera, Santa-Jiménez et al. (2009) encuentran que la mayoría de las orquídeas evaluadas en su estudio prefieren sustratos en los que se encuentran líquenes o musgos, observando que especies como Ponera striata y Epidendrum sp. se encontraban ubicadas exclusivamente en sustratos que presentaban estas coberturas. De igual manera, Tremblay et al. (1998) encontraron una relación positiva entre el porcentaje de cobertura de musgos en árboles hospederos y la presencia de L. caritensis; sin embargo, no observaron una asociación significativa entre ninguna de las 12 especies de musgo encontradas en los troncos de los hospederos y la presencia de esta especie. Estos datos sugieren que más que la asociación de la 
orquídea con determinada especie de musgo, un alto porcentaje de cobertura de musgos brindaría una mayor disponibilidad de agua para el establecimiento y persistencia de L. caritensis. Gowland et al. (2011), al igual que Tremblay et al. (1998), encontraron que las orquídeas Sarcochilus hillii, Plectorrhiza tridentata y Sarcochilus parviflorus tenían preferencia por forófitos leñosos con un alto porcentaje de cobertura por musgos. Sin embargo, en el caso de $S$. hillii y $P$. tridentata encontraron que el musgo rara vez se hallaba en el punto de establecimiento de estas especies, deduciendo que más que la acumulación de musgos en el forófito sean un reservorio de agua y nutrientes para las orquídeas, estos son un indicador de un incremento en el nivel de humedad local en el área. Gowland et al. (2011), además, apuntan la posibilidad de que un alto porcentaje de musgos refleja un nicho ecológico adecuado para el establecimiento de las micorrizas necesarias para la germinación de las orquídeas.

Factores específicos del forófito como el dap, la textura de la corteza, la presencia musgos y otras plantas, que se evaluaron en el presente estudio, tienen influencia en la distribución de las plantas epífitas. (Cornelissen y ter Steege, 1989) agregan a estos factores la porosidad, pH y composición química de la corteza.

En este estudio se observaron especies que crecieron exclusivamente en cortezas lisas o rugosas, mientras otras crecieron en ambos tipos de corteza. En un estudio realizado en la reserva forestal Gunung Manyutan al este de Java, Yulia et al. (2011) encontraron una estrecha relación entre la presencia de orquídeas y el tipo de textura de la corteza del forófito, en la que todos los árboles que contenían orquídeas poseían corteza rugosa. De igual forma, Adhikari y Fisher (2011) al estudiar los patrones de distribución de orquídeas en el Valle de Katmandú, Nepal, observaron una alta preferencia de la orquídea Rhynchostylis retusa por forófitos de corteza rugosa. Sin embargo, Gowland et al. (2011) no encontraron relación entre la presencia de las orquídeas Sarcochilus hillii, Plectorrhiza tridentata y Sarcochilus parviflorus y las características morfológicas de la corteza. Así mismo, Otero et al. (2007) no encontraron relación entre el tipo de corteza y la presencia de Psychilis monensis. Bergstrom y Carter (2008), al estudiar poblaciones de Epidendrum magnoliae, observaron cierto nivel de preferencia por árboles de Magnolia grandiflora y Quercus virginiana, los cuales poseen corteza lisa y rugosa respectivamente, sugiriendo que la estructura de la corteza no es el factor de mayor importancia para el establecimiento de E. magnoliae. Conclusión a la que también Ilegan Otero et al. (2007), al plantear que otras características pueden influenciar de forma directa la presencia de epífitas o de forma indirecta al afectar la distribución de hongos micorrícicos de los que dependen las orquídeas para su germinación.

Algunas especies como Lepanthes troxis, Stelis sp y Stelis sp2 presentaron un alto nivel de relación con el dap; entretanto, otras especies no presentan este comportamiento. El tamaño de los hospederos podría ser un factor que influencia los valores de diversidad y abundancia de orquídeas en un ecosistema. Tremblay y Velázquez (2009) observaron que la mayoría de especímenes de L. eltoroensis se encontraban en parches ubicados preferentemente en árboles con un dap promedio de $21 \mathrm{~cm}$, a pesar de que el tamaño de los troncos no mostró ser un predictor efectivo del número de orquídeas presentes en el árbol. Adhikari y Fisher (2011) dieron cuenta de una relación positiva entre el dap de los árboles hospederos y el número de especímenes de $R$. retusa, deduciendo que árboles de grandes tamaños ofrecen un mayor espacio y sustrato para el crecimiento y desarrollo de las orquídeas. Para estos autores, relictos de bosque primario pueden tener una mayor diversidad y abundancia de orquídeas que los de bosque secundario al poseer árboles de mayor edad (y por lo tanto mayores dap), posiblemente brindando una estructura boscosa desarrollada en un largo periodo de tiempo que resulta en un hábitat más complejo, extensivo y estable para las epífitas. A pesar de ello, Parra (2012) encuentra una baja correlación entre el tamaño de 
la estructura arbórea y la riqueza y diversidad de orquídeas muestreadas dentro de diferentes relictos boscosos, aunque encontró una tendencia positiva entre estas variables dentro de cada relicto examinado.

Gran parte de las orquídeas estudiadas crecieron en forófitos orientados verticalmente. Sin embargo, algunas especies lo hicieron especialmente sobre ramas o troncos con orientación horizontal e inclinada, la orientación de las ramas o troncos en los que se encontraron orquídeas da una idea del tipo de forófito y la arquitectura que este presenta. Estos factores desempeñan un papel importante debido a los niveles de regulación de la luz que puede ejercer el dosel (Yulia et al., 2011; Bergstrom y Carter, 2008). Los micrositios de los forófitos habitados por orquídeas epífitas son una consecuencia de la variación espacial por la disponibilidad de la luz (Ventre-Lespiaucq et al., 2017). Además, resaltan que las epífitas que se encuentran en los micrositios sombreados provocan en los individuos estrategias para la interceptación de la luz. También, está la capacidad de disminuir la pérdida de agua a través de las diferentes épocas del año (Bergstrom y Carter, 2008) y por las posibilidades que pueden brindar los diferentes tipos de arquitectura para el depósito de semillas y materia orgánica (Cornelissen y ter Steege, 1989).

\section{CONCLUSIONES}

La abundancia y distribución de las diferentes especies de orquídeas observadas en este estudio obedece a la compleja interrelación de los factores ambientales imperantes en la zona y, en el caso de las orquídeas epífitas, de las comunidades de forófitos establecidas, la manera en la que estos se distribuyen y sus características específicas.

Se evidencia la relación existente entre el porcentaje de cobertura de musgos en los forófitos con la abundancia y distribución de ciertas especies. Si bien es un fenómeno asociado a la humedad relativa del lugar, este factor puede estar implicado en otros procesos que facilitan el establecimiento de orquídeas.

El $73 \%$ de las especies de orquídeas registradas en este estudio se restringieron a uno de los sitios evaluados. Ello es una prueba de su estrecho intervalo de tolerancia, pues si las condiciones de ese lugar cambian es muy probable que su supervivencia se vea amenazada.

\section{AGRADECIMIENTOS}

A la Universidad de Ciencias Aplicadas y Ambientales por el apoyo en la realización de la investigación, especialmente en los trámites para los permisos correspondientes con la Corporación Regional de Cundinamarca y a Camilo Roa-Fuentes por su valiosa asesoría en los análisis multivariados.

\section{CONFLICTOS DE INTERÉS}

Los autores declaran no tener conflicto de intereses.

\section{CONTRIBUCIÓN POR AUTOR}

Ambos autores son los únicos responsables de la obra en todos los aspectos que condujeron a la elaboración de su publicación.

\section{REFERENCIAS}

Adhikari, Y. y Fischer, A. (2011). Distribution pattern of the epiphytic orchid Rhynchostylis retusa under strong human influence in Kathmandu valley, Nepal. Botanica Orientalis-Journal of Plant Science, 8(2011), 90-99.

https://doi.org/10.3126/botor.v8i0.5956

Andrade, H. y Cerda, R. (2010). Dasometría y medición de sombra. Costa Rica: Catie. 49 p. 
Atwood, J. (1986). The size of the Orchidaceae and the systematic distribution of epiphytic orchids. Selbyana, 9(1), 171-186.

Baadi, M., Landeros, J. y Cerna, E. (2008). Patrones de asociación de especies y sustentabilidad. Daena: International Journal of Good Conscience, 3(1), 632-660.

Bergstrom, B. y Carter, R. (2008). Host tree selection by an epiphytic orchid, Epidendrum magnoliae Muhl in an inland hardwood hammock in Georgia. Southeastern Naturalist, 7(4), 571-580.

https://doi.org/10.1656/1528-7092-7.4.571

Betancur, J., Sarmiento, H., Toro-Gonzáles, L. y Valencia, J. (2015). Plan para el estudio y la conservación de las orquídeas en Colombia. Bogotá: Ministerio de Ambiente y Desarrollo Sostenible, Universidad Nacional de Colombia. 336 p.

Calderón, E. (2007). Libro rojo de plantas de Colombia. Orquídeas, primera parte, vol. 6. Colombia: Eduardo Calderón Saenz editor. 828 p.

Colaboradores de LibreOffice (2016). LibreOffice Calc. The Document Foundation, Devian and Ubuntu.

Cornelissen, J. y Ter Steege, H. (1989). Distribution and ecology of epiphytic bryophytes and lichens in dry evergreen forest of Guyana. Journal of Tropical Ecology, 5, 131-150.

https://doi.org/10.1017/S0266467400003400

Davis, F., Stoms, D., Estes, J., Scepan, J. y Scott, M. (1990). An information systems approach to the preservation of biological diversity. International Journal of Geographical Information Systems 4(1), 55-78.

https://doi.org/10.1080/02693799008941529

Davis, S. y Heywood, V. (1994). Centres of Plant Diversity. A guide and strategy for their conservation. Vol. 1: Europe, Africa, South West Asia and the Middle East. Cambridge: IUCN Publications Unit. 526 p.

De La Rosa-Manzano, E., Andrade, J., Zotz, G. y Reyes-García, C. (2014). Epiphytic orchids in tropical dry forests of Yucatan, Mexico Species occurrence, abundance and correlations with host tree characteristics and environmental conditions. Functional Ecology of Plants, 209(2), 100-109.

Ding, Y., Liu, G., Zang, R., Zhang, J., Lu, X. y Huang, J. (2016). Distribution of vascular epiphytes along a tropical elevational gradient: disentangling abiotic and biotic determinants. Scientific Reports, 6(19706), 1-11.

https://doi.org/10.1038/srep19706

ESRI (2011). ArcGIS Desktop: Release 10. Redlands, Canada: Environmental Systems Research Institute. Recuperado de

https://www.arcgis.com/

Focho, D., Fonge, B., Fongod, A. y Essomo, S. (2010). A study of the distribution and diversity of the Family Orchidaceae on some selected lava flows of Mount Cameroon. African Journal of Environmental Science and Technology, 4(5), 263-273.

Forest Renewal Bc. (1999). Gap Light Analyzer: Columbia Británica, Canadá y Nueva York, Estados Unidos. Recuperado de

https://www.caryinstitute.org/science-program/our-scientists/dr-charles-d-canham/ gap-light-analyzer-gla

Gentry, A. y Dodson, C. (1987). Diversity and Biogeography of Neotropical Vascular Epiphytes. Annals of the Missouri Botanical Garden, 74(2), 205-233.

https://doi.org/10.2307/2399395

Giraldo, G. y Betancur, J. (2011). Guía de campo de las orquídeas de Santa María (Boyacá, Colombia). Serie de Guías de Campo del Instituto de Ciencias Naturales, Universidad Nacional de Colombia n. ${ }^{\circ}$ 9. Bogotá: Instituto de Ciencias Naturales, Universidad Nacional de Colombia. $188 \mathrm{p}$.

Gowland, K., Wood, J., Clements, M. y Nicotra, A. (2011). Significant Phorophyte (substrate) Bias is not Explained by Fitness Benefits in Three Epiphytic Orchid Species. American Journal of Botany, 98(2), 197-206.

Hágsater, E. (2001). Icones Orchidacearum Fascicle 4. The genus epidendrum Part 3. "Third Century of New Species in Epidendrum". México D. F.: Eric Hágsater y Luis Sánchez Saldaña (eds.). 72 p.

Hágsater, E. (2009). Icones Orchidacearum Fascicle 12. The genus epidendrum Part 8. "Species New \& Old in Epidendrum". México D. F.: Eric Hágsater y Luis Sánchez Saldaña (eds.). 72 p.

Hágsater, E. (2016). The genus epidendrum Part 11: Species New \& Old in Epidendrum. Icones 
Orchidacearum Fascicle, 15(2). México D. F.: Eric Hágsater y Luis Sánchez Saldaña (eds.). 72 p.

Hammel, B., Grayum, M., Herrera C. y Zamora N. (2003). Manual de plantas de Costa Rica. Vol. III, Monocotiledóneas (Orchidaceae-Zingiberaceae). Missouri: Missouri Botanical Garden Press. 884 p.

Hammer, O., Harper, D. A. T. y Ryan, P. D. (2001). PAST: Paleontological Statistics Software for education and data analysis. Paleontologia Electronica, $4(1), 9$.

Ibisch, P. L., Nowicki, C., Müller, R. y Araujo, N. (2002). Methods for the assessment of habitat and species conservation status in data-poor countries - case study of the Pleurothallidinae (Orchidaceae) of the Andean rain forests of Bolivia. En: R. Bussmann y S. Lange (eds.), Proceedings of the First International Congress "Conservation of Biodiversity in the Andes and the Amazon Basin (pp. 24-28). Cuzco, Perú.

Instituto de Ciencias Naturales de la Universidad Nacional de Colombia (2018). Herbario virtual. Recuperado de

http://www.biovirtual.unal.edu.co/es/colecciones/ search/plants/

IUCN/SSC Orchid Specialist Group (1996). Status Survey and Conservation Action Plan: Orchids. Gland Swiza y Cambridge, Reino Unido: IUCN Publications Services Unit. 153 p.

Jardín Botánico de Bogotá José Celestino Mutis (2018). Herbario JBB en línea. Recuperado de

http://colecciones.jbb.gov.co/herbario/especimen/ simple

Johansson, D. (1974). Ecology of vascular epiphytes in West African Rain Forest. Acta Phytogeographica, 59, 1-136.

Luer, C. A. (1986). Icones Pleurothallidinarum I. Systematics of the Pleurothallidinae. Monographs in Systematic Botany. Sarasota: Missouri Botanical Garden. Arcade Lithographing Corp. 81 p.

https://doi.org/10.5962/bhl.title.149317

Luer, C. A. (2004). Icones Pleurothallidinarum XXVI. Pleurothallis subgenus Acianthera and three allied subgenera. A second century of new species of Stelis of Ecuador. Epibator, Ophidion, Zootrophion. Missouri: Missouri Botanical Garden Press. 265p.
Luer, C. A. y Thoerle, L. (2012). Icones Pleurothallidinarum XXXII. Lepanthes of Colombia (Orchidaceae). Monographs in Systematic Botany from the Missouri Botanical Garden. Missouri: Missouri Botanical Garden Press. 298 p.

Mejía, H. y Pino, N. (2010). Diversidad de orquídeas epífitas en un bosque húmedo tropical (bh-t) del departamento del Chocó, Colombia. Acta Biológica Colombiana, 15(2), 37-46.

Missouri Botanical Garden (2018). Tropicos. Recuperado de

http://www.tropicos.org/SpecimenSearch.aspx

Orejuela-Gartner, J. (2015). Orchids of the cloud forests of southwestern Colombia and opportunities for their conservation. European Journal of Environmental Sciences, 2(1), 19-32.

Ortiz, P. (1995). Orquídeas de Colombia. Segunda edición. Bogotá: Corporación Orquideología. 320 p.

Ortiz, P. y Uribe-Vélez, C. (2007). Galería de orquídeas de Colombia. Bogotá: Asociación Bogotana de Orquideología.

Phal, J. (2018). Internet Orchid Species Photo Encyclopedia. En

http://orchidspecies.com/

Otero, T., Aragón, S. y Ackerman, J. (2007). Site Variation in Spatial Aggregation and Phorophyte Preference in Psychilis monensis (Orchidaceae). Biotropica, 39(2), 227-231.

https://doi.org/10.1111/j.1744-7429.2006.00258.x

Parra, E. (2012). Efecto de los patrones del paisaje sobre la diversidad de orquídeas de bosques nublados del Valle del Cauca (tesis de maestría, Biología). Universidad Nacional de Colombia, Facultad de Ciencias, Bogotá, Colombia. 92 p.

Revilla, N., Moscoso, D. y Nauray, W. (2003). Problemática de la familia Orchidaceae en el Valle Sagrado de los Incas. Lyonia, 4(1), 19-24.

Roberts, D. y Brummitt, N. (2006). The Influence of Geometric Constraints on the Colonisation, Speciation and Range Expansion of Orchids. En: Proceedings of the XVII AETFAT Congress (361-372 pp). Addis Ababa: Addis Ababa University Press.

Santa-Jimenez, N., García, T. y Gómez, G. (2009). Estructura y composición de la comunidad de orquídeas 
en dos zonas de la reserva natural "La Montaña del Ocaso" Quimbaya-Quindío. Revista de Investigaciones de la Universidad del Quindío, 19, 122-134.

Solano, R., Cruz, G., Martinez, F. y Lagunez, L. (2010). Plantas utilizadas en la celebración de la Semana Santa en Zaachila, Oaxaca Mexico. Polibotanica, 29(2010), 263-279.

Sugden, A. y Robins, R. (1979). Aspects of the ecology of vascular epiphytes in Columbian cloud forests. I. The distribution of the epiphytic flora. Biotropica, 11(3), 173-188.

https://doi.org/10.2307/2388037

Swiss Orchid Foundation (2016). Swiss Orchid Foundation at the Herbarium Jany Renz. Recuperado de https://orchid.unibas.ch/index.php/en/database-search/ advanced-search

Tian, H. y Xiang, F. (2008). Elevational diversity patterns of orchids in Nanling National Nature Reserve, northern Guangdong Province. Biodiversity Science, 16(1), 75-82.

Tremblay, R., Zimmerman, J., Lebrón, L., Bayman, P., Sastre, I., Axelrode, F. y Alers-Garcia, J. (1998). Host specificity and low reproductive success in the rare endemic Puerto Rican orchid Lepanthes caritensis. Biological Conservation, 85, 297-304.

https://doi.org/10.1016/S0006-3207(97)00163-8
Tremblay, R. (2008). Ecological correlates and shortterm effects of relocation of a rare epiphytic orchid after Hurricane Georges. Endangered Species Research, 5, 83-90.

Tremblay, R. y Velazquez, J. (2009). Circular distribution of an epiphytic herb on trees in a subtropical rain forest. Tropical Ecology, 50(2), 211-217.

Ventre-Lespiaucq, A., Delgado, J. A., Ospina-Calderon, N., Otero, J. T., Escudero, A., Sanchez, M. A., Balaguer, L. y Flanagan, N. S. (2017). A tropical epiphytic orchid uses a low light interception strategy in a spatially heterogeneous light environment. Biotropica, 49(3), 318-327.

Wagner, K., Mendieta-Leiva, G. y Zotz, G. (2015). Host specificity in vascular epiphytes: a review of methodology, empirical evidence and potential mechanisms. AoB Plants, 7, 1-25.

https://doi.org/10.1093/aobpla/plu092

Yulia, N., Budiharta, S. y Yulistyarini, T. (2011). Analysis of epiphytic orchid diversity and its host tree at three gradients of altitudes in Mount Lawu, Java. Biodiversitas, 12(4), 225-228.

https://doi.org/10.13057/biodiv/d120407

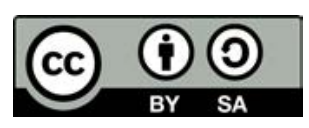

Colombia Forestal •ISSN 0120-0739 • e-ISSN 2256-201X • Bogotá-Colombia • Vol. 23 No. 1 • Enero-Junio de $2020 \bullet$ pp. 5-19 